\title{
Labour ward incidents and potential claims - lessons learned from research
}

\author{
Dr Brenda Ashcroft
}

\begin{abstract}
This paper provides an insight into the underlying factors involved in potential cerebral palsy and/or shoulder dystocia claims. The research was undertaken to identify the root causes of 37 cases of birth asphyxia in term infants severe enough to warrant admission to neonatal care units in the north-west of England between 2001 and 2002. All available staff $(n=93)$ providing care during critical periods were interviewed by the author using the cognitive interviewing technique. These included 81 midwives, two consultant obstetricians, eight registrars and two senior house officers. An expert panel consisting of consultant obstetricians, midwives, a consultant neonatologist and the researcher applied the Bolam test to identify instances where care had been substandard and injury caused as a result. Although the cases were often complex, covering more than one shift and over more than one stage of labour, the most dangerous time appeared to be during the night shift (19 cases, 51\%), followed by the evening shift (13 cases, 35\%) and then the day shift (five cases, 14\%). The main problems include: failure to respond appropriately to signs of fetal hypoxia (26 cases, 70\%); undiagnosed obstruction (22 cases, 59\%), which was broken down into failure to identify cephalopelvic disproportion (13 cases, 35\%); and shoulder dystocia (nine cases, 24\%). Delayed resuscitation of the infant occurred in 26 cases (80\%), and in 18 cases (49\%) there was excessive and inappropriate use of Syntocinon. All cases involved human error, either through a delay or failure to take action, or taking inappropriate action. However, these were all underpinned and perpetuated by system and cultural errors present in the labour wards, such as allowing unsupported and inexperienced personnel to work in a position for which they lacked the necessary skill and experience. This was perpetuated by the customary practice of using unsupervised junior medical staff in a first on-call position for complications, and also of failing to sustain safe midwifery staffing levels. This in turn prevented support for more inexperienced staff. Consequently, when inexperienced midwives and obstetricians were left unsupervised in charge of complicated cases, it created accidents waiting to happen. When unsupervised and inexperienced paediatricians attended the birth of an asphyxiated infant, the child's condition deteriorated further when they were unable to resuscitate it. If such system and cultural errors as these are not rectified, the current high rate of damaged babies is likely to continue.
\end{abstract}

\section{Introduction}

A recent report has suggested that an average claim for a brain-damaged child is between $\mathcal{A}^{3} 3.5$ and $£, 5$ million and that the incidence of hypoxic ischaemic encephalopathy (HIE) with the potential to result in one of these claims is one to three per 1000 live births. Consequently, hospitals with a yearly rate of 4800 deliveries may expect to have five cases of moderate to severe HIE per year. ${ }^{1}$ Despite an obvious moral need to reduce this rate of harm and curb the financial drain of litigation on the NHS, it appears that maternity trust Boards are still giving insufficient priority to safety. ${ }^{2}$ Although previous national and regional confidentiality enquiries 3,4 have reported that substandard care has been a major cause of mortality, they have lacked the necessary in-depth investigations to identify root or underlying causes of errors made by staff. Similarly, it is generally accepted that incident reporting schemes, such as that organized by the National Patient Safety Agency (NPSA) are subject to low levels of reporting. As there is little published material on the underlying reasons for substandard care, ${ }^{5}$ this area is ripe for research.
This article describes research involving an in-depth investigation to identify the root causes of 37 cases of birth asphyxia severe enough to warrant admission to a neonatal care unit. In 30 cases, the infants were diagnosed to have some degree of HIE, which could result in future cerebral palsy claims. Two infants in these cases and two others also suffered from Erb's palsy. Although the remaining five cases were likely to be near-misses, they were not excluded from the research as it is accepted that near-misses and patient safety incidents share the same underlying causes. ${ }^{6}$

\section{Methods}

The study was undertaken by the author between February 2001 and March 2002 in the labour wards of seven maternity units in the north-west of England. The Trusts were geographically situated to provide a range of experiences and all complied to level one of the standards set at that time by the Clinical Negligence Scheme for Trusts. With the assistance of midwifery managers and neonatal unit staff, at least five of the most recent admissions to the neonatal unit for severe birth asphyxia in term infants $(n=37)$ were selected from each unit.

All available members of staff providing care during critical periods in these cases were interviewed using the cognitive interviewing technique. This technique has been shown to uncover up to $63 \%$ more information than 
traditional interviewing techniques, ${ }^{7}$ and the NPSA now recommend it for use with their Root Cause Analysis Tool. ${ }^{8}$ In total, 93 members of staff were interviewed, including 81 midwives, two consultant obstetricians, eight registrars and two senior house officers (SHOs).

Extensive case descriptions were generated from the interviews, together with information contained in the casenotes and cardiotocograph (CTG) recordings. Background information was also provided from an observational study in the same units the previous year, some of which has been reported. ${ }^{9}$ An expert panel applied the Bolam test to identify where the standard of care fell below an acceptable level. The panel included two consultant obstetricians, a consultant neonatologist and neonatal midwife, a consultant midwife and a midwifery risk manager, in addition to the researcher. Thematic analysis was undertaken using the protocol from the Clinical Risk Unit (CRU) and Association of Litigation and Risk Management (ALARM) for analysing adverse incidents. $^{10}$

\section{Results}

Figure 1 shows a breakdown of the 37 cases.

\section{When the problems arose and type of problem}

Although the cases were often complex with errors occurring over more than one shift, more errors occurred during the night shift (in 19 cases, 51\%). The evening shift also involved a significant number of errors (13 cases, 35\%), but least of all occurred during the early shift (five cases, 14\%). One reason for this appeared to be the lack of adequate on-site expert medical assistance after 17:00 hours when the on-call system began. Delays occurred during this time when labouring women with complications waited in turn to be seen by the registrar (the most senior available obstetrician), as the on-call consultant worked from home. Midwifery staffing levels were often inadequate during these shifts, especially during periods of sickness and holidays, as the unit's staffing levels rarely ever achieved the recommended minimum staffing levels. ${ }^{9,11}$

Although errors often occurred during more than one stage of labour, the majority occurred during the first stage (in 31 cases, 84\%). In 19 cases (51\%), they occurred during the second stage of labour and in 27 cases (73\%) they followed delivery. The categories of errors (Figure 2) included: failing to respond to fetal hypoxia 26 cases (70\%); undiagnosed obstruction 22 cases (59\%), which in turn was due

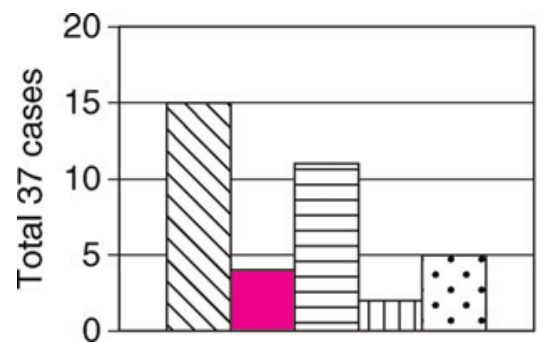

$\triangle$ Poor prognosis

$\square$ Potentially poor prognosis

日 Level of damage uncertain

प] Erb's Palsy

$\square$ Probable near-miss

Figure 1 Outcome of 37 cases of severe birth asphyxia. Fifteen cases involved infants with a poor prognosis (moderate or severe hypoxic ischaemic encephalopathy [HIE]); four cases HIE/potentially poor prognosis (two also had Erb's palsy); 11 cases mild HIE or equivalent; two cases Erb's palsy; five probable near-misses

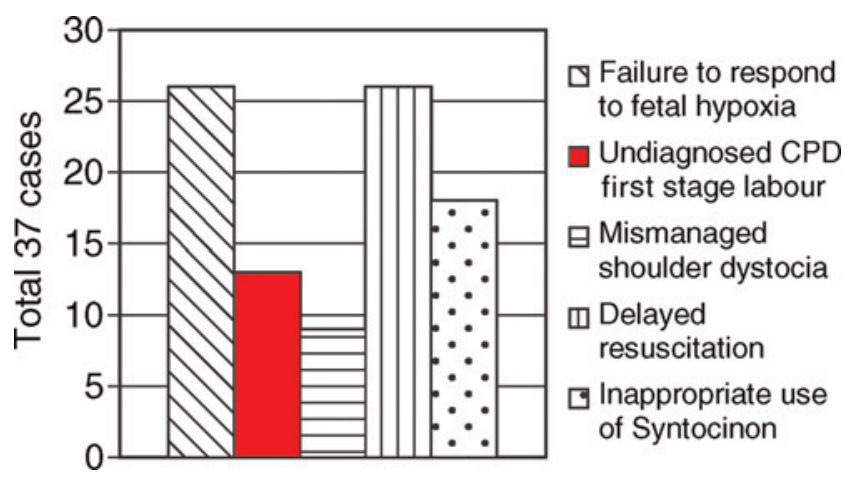

Figure 2 Main causes of error

to unrecognized cephalopelvic disproportion (CPD) in the first stage of labour in 13 cases (35\%); and mismanaged shoulder dystocia during the second stage of labour in nine cases (24\%). Errors due to delays in providing adequate infant resuscitation following delivery occurred in 26 of the 37 cases (70\%). In 18 cases (49\%), there was an inappropriate use of Syntocinon, either due to an unwarranted or excessive use, which caused traumatic moulding of the fetal head in four cases of undiagnosed CPD.

\section{Underlying causes of error}

Although failing to take action, taking inappropriate or delayed action were all due to human error, it was discovered that they were underpinned and perpetuated by system and cultural errors in each of the maternity units, for example heavy workloads with an insufficient number of midwifery staff to manage the cases safely. This occurred in 26 cases $(70 \%)$, and in 10 out of the 26 cases there was a reduction of at least one midwife from the establishment numbers. In 10 of these 26 cases there was also a poor skill-mix (i.e. disproportionate numbers of inexperienced staff). Therefore delays occurred when complications requiring medical assistance were either not identified or dealt with promptly. One midwife, qualified for only six weeks and working unassisted when a shoulder dystocia occurred, commented:

'You're on the main delivery unit and you've got an obstetric emergency and you can't get help.'

When such problems corresponded with occasions when junior doctors were acting in a first on-call position for complications or when there were restricted numbers of medical staff available (such as during the evening and night), delays in decision-making and providing emergency treatment became inevitable. The difficulties facing a solitary registrar and house officer during one night was described on one occasion by a midwife:

'The registrar had a really dreadful night. She had possibly another two or three emergency sections. She looked absolutely exhausted and, was, I think, at the end of her tether.'

Support for junior midwives and midwives inexperienced in labour ward work was not always possible during intensive workloads, which was epitomized in the following comment by a midwife qualified for one year:

'We've got a lot of junior staff who need guidance... and sometimes you can't get it.'

Working beyond their level of competence and experience without support was a common and worrying experience, 
illustrated by the following comment by a midwife qualified for 15 years, but inexperienced in labour ward work,

'It's very scary. . . very scary for the mother and very scary for the midwives.'

The system of care provided in the labour wards contained cultural and hierarchical issues that provoked error in staff in 35 cases (95\%). In 29 cases (78\%), it occurred through a failure of senior staff to provide assistance for junior and inexperienced obstetricians and paediatricians. The culture also discouraged the latter from directly seeking it through fear of being labelled lacking in ability or not being able to cope. This also affected registrars, for despite a clear (although not overtly voiced) need for assistance when ringing the on-call consultant regarding very complicated cases or excessive workloads, the latter rarely attended during weekends and the nights. One experienced registrar commented on his disappointment when the on-call consultant failed to attend during a very complicated case:

'I mean, that was a high-risk case and when I'm a consultant

I'm going to be in. If my registrar rings me up with a case like that - I'm there.'

The position was similar for junior paediatricians, as a midwife qualified for eight years explained:

'The first week they're supposed to be supervised by the registrar, but in reality it doesn't happen.'

Junior paediatricians were not provided with the same degree of training in infant resuscitation as midwives and therefore their skills and knowledge were often deficient. Despite this fact, in 17 cases (46\%) they remained unsupervised during the birth of a severely asphyxiated infant. When they were unable to resuscitate the child a delay occurred before the registrar's assistance was forthcoming. When it can be anticipated that advanced methods of resuscitation will be required at a birth the appropriate level of skilled practitioner should be in attendance, but an experienced midwife (qualified for 17 years) confirmed that this was not always the case:

'We've discussed having junior paediatricians with problem cases for a long time. Very often you 'fast bleep' [emergency call for senior staff] a paediatrician and somebody gets there that can't do any more than you can. It's not fair to anybody'

Communication problems regarding different practices for summoning medical assistance were also evident in the labour wards. One midwife qualified for two years commented:

'I'm not really sure who to call. I think we're supposed to call the SHO first, but some G grades [senior midwives] call the reg.'

However, calling an SHO first often added a delay of at least 30 minutes when remedial action such as Caesarean section was necessary. This problem was most common during the night. One midwife qualified for two years commented on one situation that occurred during the night:

'You can ask the reg, but they don't always come. They send the SHO and tell you they'll only come if the SHO can't manage.'

Differences of opinion regarding what action to take were also evident between midwives and obstetric registrars in 17 cases (46\%), with no mechanism in place to support resolution of the dispute. One midwife qualified for three years commented:

'You go through all this training as a midwife to be taught this is abnormal and you're meant to get somebody in. It's like you're recognizing this is abnormal, but at the same time the doctors are saying everything's OK.'
The hierarchical structure operating in the labour ward invariably resulted in midwives' judgement being overruled, despite some of them having more experience. As one midwife explained:

'I've been a midwife for 30 years, very often a registrar might have been doing obstetrics for two years. You can't compare the two, can you?'

Despite a professional responsibility to protect the wellbeing of those in their care, ${ }^{12}$ midwives found it difficult to report the case to the consultant following a difference of opinion, especially during the night. This was due to fear of damaging the working relationship with the registrar and/or fear of an unfavourable reaction from the consultant. As one midwife qualified for 30 years reported:

'It's alright saying with hindsight you can call a consultant. There are some that would be very sympathetic [to you], but there are certain consultants who would haul you over the boards for something like that.'

Another midwife, qualified for 22 years described what she felt was the futility in doing so:

'Some of the consultants would just say, "The registrar's seen her. I'm happy with that."'

Experience and skill are vital for the safe management of obstetric complications, yet lack of experience and guidance was evident in many cases. One senior registrar compared past practices with those of today, which he believed predisposed to human error:

'... the consultant used to teach how to do such things as shoulder dystocia and the use of forceps, but that doesn't happen any longer... the junior doctors were supervised and not just allowed to undertake things like instrumental deliveries on their own.'

Lack of skill, knowledge and experience was particularly evident with regard to signs of obstruction in the first stage of labour resulting from either malposition or CPD. In all but one case it resulted in inappropriate and prolonged use of Syntocinon, causing one member of the expert panel to comment:

'This registrar seems to want a vaginal delivery at any cost.'

When the research was being conducted the National Sentinel Caesarean Section Audit was in progress ${ }^{13}$ and there appeared to be a drive to reduce the number of unnecessary Caesarean sections. Consequently, once labour had begun many obstetricians appeared to have a reluctance to intervene and perform Caesarean section, which contrasted with what appeared to be the generous provision of Caesarean sections when planned beforehand.

In the labour wards an over-liberal use of Syntocinon became apparent, which resulted in practitioners resorting to its use as soon as labour became abnormal or arrested, without first considering the underlying reasons. Failure to identify the underlying problem and the associated dangers may be due to a reduced emphasis on antenatal detection of $\mathrm{CPD}$, which has resulted in lowering its profile and subsequent identification. Although it has generally been accepted that CPD can only be diagnosed in women having a first baby following trial of labour with adequate uterine contractions, ${ }^{14}$ in this study the signs were poorly recognized by members of staff. In all cases practitioners were aware that there were problems, but not precisely what they were. Diagnosis was hindered when 
inexperienced midwives and doctors were left unsupported in charge of these cases, either during busy periods or during the night when fewer medical staff were available. The ensuing prolonged labours also resulted in a number of different caregivers (both midwives and obstetricians) when the labours occurred over more than one shift. The 'whole picture' consequently became indistinct as the practitioners involved concentrated on resolving immediate problems. In these cases the consequences for the infants were either cerebral trauma through prolonged use of Syntocinon, excessive moulding of the fetal skull and/or inappropriate or repeated use of instruments. This resulted in one member of the expert panel to sum up that the baby was obviously,

'Like a square peg being pushed through a round hole.'

Inappropriate use of Syntocinon was not confined to situations involving slow progress in labour. For example, it arose in one case of early labour in which there had been episodes of reduced fetal movements, fetal bradycardia, thick fresh meconium, with the CTG recording showing reduced variability and early decelerations of the fetal heart rate. In this case its use was clearly contraindicated, yet the midwife reported that the consultant had ordered:

'Start Synto and see what happens.'

In another case while awaiting the registrar following an episode of prolonged fetal bradycardia of $66 \mathrm{bpm}$, the midwife changed the empty bag of Syntocinon to a full one and continued to run the infusion at a maximum rate of $96 \mathrm{~mL} /$ hour. Such grossly inappropriate uses of Syntocinon led one member of the expert panel to ask in disbelief:

'Is Synto the answer to a poor trace?'

Despite unit protocols and guidelines giving instructions for Syntocinon regimens, none of them offered advice on contraindications and risks, or the special precautions that should be taken with its use. Instead, an over-familiarity with its use appears to have led to a diminished appreciation of the risks involved.

\section{Conclusion}

Many problems occurred through all stages of labour due to human error, resulting in failures to take action, delays in taking action or in taking inappropriate action. However, these were directly initiated by such system failures as allowing unsupported personnel to work in a position for which they lacked sufficient skills, training, knowledge or experience. Such problems were encouraged by the customary practice of using junior medical staff in a first on-call position and having reduced medical cover during evenings and the night. This was situated within a culture where openly seeking assistance appeared to be frowned upon. A failure to sustain minimal midwifery staffing levels similarly prevented support and guidance being available for junior and inexperienced midwives, and when complicated cases were managed by inexperienced and unsupported staff, especially during heavy workloads, it resulted in accidents waiting to happen. Such deficiencies, which allow mistakes to occur through lack of knowledge and experience, include the failure to detect abnormalities such as fetal hypoxia and CPD. Mistakes were often exacerbated by erroneous and excessive use of Syntocinon at times in an apparent effort to achieve vaginal delivery once labour had begun. Birth hypoxia arising from such errors was then frequently prolonged when the least experienced personnel were sent to resuscitate these asphyxiated infants. Such underlying causes of error highlight an urgent need for significant changes in the system used in labour wards. If this does not happen the high number of damaged babies is likely to continue at the present rate.

\section{Funding}

The research received original funding from the NHS Executive North West R\&D, the North West Lancashire Health Authority and the National Patient Safety Agency.

\section{Ethical approval}

The North West multiresearch ethics committee approved the study, as did each of the seven trust's local research ethics committees. Permission was gained from leads of midwifery and obstetrics in each unit.

\section{References}

1 Royal College of Obstetricians and Gynaecologists written evidence for Safer Births. Everybody's Business: An Independent Inquiry into the Safety of Maternity Services in England (Chaired by Onora O’Neill). London: King's Fund, 2008: 88

2 Health Care Commission written evidence for Safer Births. Everybody's Business: An Independent Inquiry into the Safety of Maternity Services in England (Chaired by Onora O'Neill). London: King's Fund, 2008: 73

3 Confidential Enquiry into Stillbirths and Deaths in Infancy (CESDI). 4th Annual Report 1997. Maternal and Child Health Research Consortium. London: Department of Health, 1997

4 Confidential Enquiry into Stillbirths and Deaths in Infancy (CESDI). Annual Report for 1 January-31 December 1993. Part 1: Summary of Methods and Main Results. London: Department of Health, 1995

5 Ennis M, Vincent CA. Obstetric accidents: a review of 64 cases. BMJ 1990; 300: 1365-7

6 van der Shaff TW. Development of a near miss development system at a chemical process plant. In: van der Shaff TW, Hale AR, Lucas DA, eds. Near Miss Reporting as a Safety Tool. Oxford: Butterworth-Heinemann, 1991

7 Fisher RP, Geiselman RE, Amador M. Field test of the Cognitive Interview: enhancing the recollection of actual witnesses of crime. J Appl Psychol 1989; 74: 722-7

8 National Patient Safety Agency. Root Cause Analysis Tool. London: NPSA, 2003. See http://www.rca@npsa.nhs.uk (last checked 20 April 2005)

9 Ashcroft B, Elstein M, Boreham N, Holm S. Prospective semi-structured observational study to identify risk attributable to staff deployment, training and updating opportunities for midwives. BMJ 2003; 327: 564-85

10 Clinical Risk Unit and Association of Litigation and Risk Management. A Protocol for the Investigation and Analysis of Clinical Incidents. London: University College London and the Royal Society of Medicine, 1999

11 Royal College of Obstetricians and Gynaecologists/Royal College of Midwives. Towards Safer Childbirth - Minimum Standards for the Organisation of Labour Wards. Report of a Joint Working Party. London: Royal College of Obstetricians and Gynaecologists, 1999

12 Nursing and Midwifery Council. The Code: Standards of Conduct, Performance and Ethics for Nurses and Midwives. London: NMC, 2008:1

13 Royal College of Obstetrics and Gynaecology. National Sentinel Caesarean Section Report - October 2001. London: RCOG Clinical Effectiveness Support Group, 2001. See http://www.rcog.org.uk/ resources/public/pdf/nscs_audit.pdf (last checked 20 April 2005)

14 Nisrat H, Warda A. X-ray pelvimitry reappraisal. Clin Exp Obstet 1991; 18: 27-33 